\title{
WAVES OF EXCITATIONS IN HETEROGENEOUS ANNULAR REGION, ASYMMETRIC ARRANGEMENT
}

\author{
ANDRÁS VOLFORD, PETER L. SIMON and HENRIK FARKAS \\ Department of Chemical Physics \\ Technical University \\ Budapest H-1521, Hungary \\ E-mail:volford@phy.bme.hu,simonp@phy.bme.hu,farkas@phy.bme.hu
}

\begin{abstract}
This paper deals with the propagation of waves around a circular obstacle. The medium is heterogeneous: the velocity is smaller in the inner region and greater in the outer region. The interface separating the two regions is also circular, and the obstacle is located eccentrically inside it. The different front portraits are classified.
\end{abstract}

1. Introduction. Waves in different fields of natural sciences can be described by partial differential equations which have solutions of the form

$$
u(t, r)=A(r) f(t-S(r)) .
$$

Here $A$ is the amplitude, $f$ is the phase, and $S$ is the eikonal. There are several equations having this type of solution; e.g. wave equation, Hamilton-Jacobi equation [1], reactiondiffusion equation $[5,10,12,13]$, heat equation $[4,3]$.

The process of propagation can be described without involving the amplitude. In the geometric theory of waves we can define rays and fronts, we can establish the evolution of fronts provided that the velocity is known $[2,15]$. From a mathematical point of view, this approach is flourishing, and produces a lot of interesting results (see e.g. [7, 14] and the other articles of this volume).

The first application of the geometrical approach to biology goes back to Wiener and Rosenblueth [16]. They constructed a model to the propagation of excitations in nerve system and cardiac muscle.

Chemical waves are very similar to the waves of excitations in biology. A simple analogue which illustrates the most striking character of chemical waves is the process of

1991 Mathematics Subject Classification: Primary 46C20; Secondary 32G81.

The authors are grateful to grants CARNET COPERNICUS Project PL 96-2168, OTKA T-017041, FKFP 0287/1997, OTKA F-22222 for supporting this research.

The paper is in final form and no version of it will be published elsewhere. 
prairie fire. Starting from an initial burning front, the process is easily predicted if the velocity is known.

Chemical waves rotating around an obstacle (chemical pinwheels) had been observed experimentally by Noszticzius and his co-workers $[9,8,11]$. Those may have importance in understanding of some characteristics of a heart disorder, 'flutter' $[8,16]$.

Simple mathematical models of chemical waves rotating around an obstacle in a homogeneous medium (the velocity is constant) were described by several authors, e.g. $[2,9,14,16]$. The case of heterogeneous annular region (the obstacle surrounded by an inner slow and an outer fast region) was investigated experimentally and theoretically [8]. Here we describe wave propagation in a heterogeneous asymmetric annular region (the obstacle is not concentric with the interface). The arrangement and notations are depicted in Fig. 1.

The paper is organized as follows. In Section 2 we describe the model and the problem to be investigated in exact terms. In Section 3 we collect some important consequences of the geometrical theory of waves to the case of heterogeneous medium (where the velocity is piecewise constant). We deal with the properties of extremals, and introduce the local break point dynamics which is a tool for the investigation of wave front evolution in heterogeneous media. Section 4 contains the main results. The stationary wave fronts are calculated in the different cases (according to the position of the obstacle and the value of the refractive index).

2. The model. In this section we determine formally the region in which our waves evolve, and the velocity of propagation. Then we formulate the most important notions and the problem under investigation.

Let us denote by $\mathcal{S}(r, P)$ the circle with radius $r$ centred at $P$, and by $\mathcal{B}(r, P)$ the open disk with radius $r$ centred at $P$.

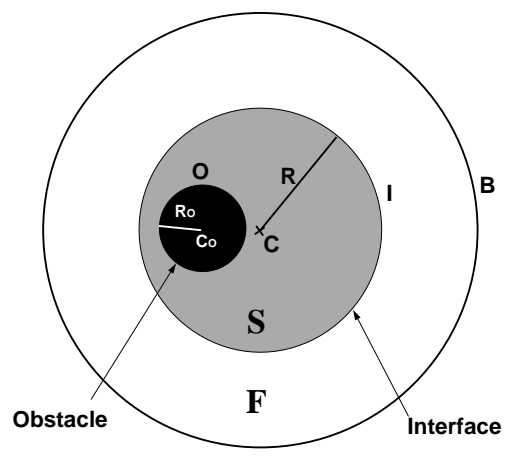

Figure 1: Asymmetric arrangement. The circular obstacle $O$ with the centre at $C_{O}$ and radius $R_{O}$ is surrounded by a heterogeneous medium. The circular interfacial boundary $I$ of radius $R$ and centre $C$ separates an inner 'slower' annular region $S$ (with propagation velocity $v_{1}$ ) and an outer 'faster' annular region $F$ (with propagation velocity $v_{2}>v_{1}$ ). The reactor $V$, where the waves propagate, is bounded by the circle $B$. 
Definition 1. Let $C$ and $C_{O}$ be points on the plane and $R_{O}<R<R_{B}$ be positive numbers for which $R-R_{O}$ is greater than the distance of $C$ and $C_{O}$. The circle $O:=$ $\mathcal{S}\left(R_{O}, C_{O}\right)$ is called obstacle, $I:=\mathcal{S}(R, C)$ is called interface (or interfacial boundary), $B:=\mathcal{S}\left(R_{B}, C\right)$ is called (outer) boundary. Let $S:=\mathcal{B}(R, C) \backslash \mathcal{B}\left(R_{O}, C_{O}\right), F:=B \cup$ $\mathcal{B}\left(R_{B}, C\right) \backslash \mathcal{B}(R, C)$ and $V:=S \cup F$. (See Fig. 1.)

DEFINITION 2. Let $v_{1}<v_{2}$ be positive numbers. We define the velocity as the function $v: V \rightarrow \mathbf{R}$

$$
v(x):= \begin{cases}v_{1} & \text { if } x \in S \\ v_{2} & \text { if } x \in F .\end{cases}
$$

Remark that if a part of the path lies on the interfacial boundary, then we should insert the greater velocity for that part due to Fermat's principle.

Definition 3. Let $f_{0} \subset V$ be a straight segment, the endpoints of which are on $O$ and $B$ respectively; this will be referred to as initial front.

Definition 4. Let $G$ be the set of piecewise smooth curves in $V$ for which the following two conditions hold:

1. The starting point of the curve is on $f_{0}$.

2. The curve can intersect $f_{0}$ only in positive direction, that is a small arc of the curve after the intersection point is on the local positive side. (The points on the local positive side can be obtained from the points of $f_{0}$ by a small anticlockwise rotation around $C_{O}$.)

These curves will be called admissible curves.

The assumption that $f_{0}$ is a straight segment is only a technical condition. Assumption 2 in Definition 4 ensures that starting from the initial front the propagation is allowed only in the anticlockwise direction, thus the front rotates around the obstacle, see Fig. 2.

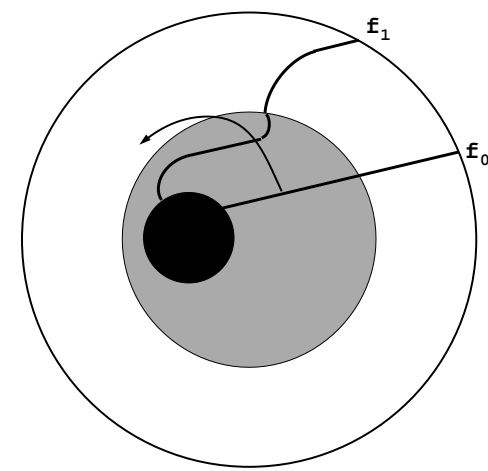

Figure 2: The initial front $f_{0}$ is a line segment. The front moves in the anticlockwise direction.

DEFINITION 5. Let $g \in G$ be an admissible curve given in arclength parametric form; the length of $g$ is denoted by $l(g)$. Let us denote by $\tau(g)$ the propagation time along $g$, that is

$$
\tau(g)=\int_{0}^{l(g)} \frac{1}{v(g(s))} d s .
$$


Definition 6. A simple closed curve surrounding the obstacle in $V$ is called minimal loop if there is no other closed curve surrounding the obstacle $O$ having less propagation time. A piecewise smooth curve in $V$ is called Fermat ray or extremal if there is no other piecewise smooth curve with same endpoints having less propagation time.

In our model the front propagation is determined by Fermat's principle, therefore the fronts are defined as the level curves of the following eikonal.

Definition 7. Let $\sigma: V \rightarrow \mathbf{R}^{+}$be the eikonal, that is

$$
\sigma(P)=\inf \{\tau(g): g \in G, g(l(g))=P\} .
$$

Let the wave front at time $t$ be

$$
f_{t}=\{P \in V: \sigma(P)=t\}
$$

The aim of this paper is to determine the shape and evolution of the fronts $f_{t}$. It turns out that after a transient interval, the process will be periodic in time with period $T$, that is an 'asymptotic' front rotates around the obstacle periodically. During a round the shape of the front changes (not as in the symmetric case). Our aim is to determine this asymptotic front portrait. The fronts are orthogonal to the extremals, therefore in order to get the shape of the fronts it is enough to determine the extremals.

When determining the extremals, certain points of the fronts (the "leading points") play a distinguished role.

Definition 8. The point $P$ of the front $f_{t_{0}}$ is called a leading point if there exists $t>t_{0}$ such that the set of points $Q \in f_{t}$ for which there exist an extremal with endpoints $P$ and $Q$ and with length $t-t_{0}$ has positive measure.

In previous works $[9,8,15]$ we applied the geometric theory based on Fermat's principle to simpler cases. If the medium is homogeneous $\left(v_{1}=v_{2}\right)$, the relevant family of Fermat rays are the forward tangential half-lines to the obstacle's boundary. These rays determine the asymptotic front lines which are just the involutes of the obstacle's boundary $[9,15,16]$. In [8] the medium was supposed to be heterogeneous, but the arrangement had circular symmetry (in the present setting it is the special case $C=C_{O}$ ). For this symmetric arrangement it turned out that there were two relevant families of rays: obstacle generated rays and interface generated rays.

1. Obstacle generated rays depart anticlockwise from the obstacle tangentially and continue to the outer region $(F)$ according to Snell's law of refraction $\left(\frac{v_{1}}{v_{2}}=\frac{\sin \alpha_{1}}{\sin \alpha_{2}}\right)$.

2. Interface generated rays depart anticlockwise from the interfacial boundary tangentially to the outer region $(F)$ and with the critical angle $\alpha_{\mathrm{cr}}$ of total reflection into the inner region $\left(\sin \alpha_{\mathrm{cr}}=v_{1} / v_{2}\right)$.

That is, two leading points generate the rays determining the asymptotic fronts, one runs around the obstacle's boundary with velocity $v_{1}$, while the other runs around the interfacial boundary $I$ with velocity $v_{2}$. The competition of these leading points determines the asymptotic front in the following way: if the angular velocity $\omega_{1}=v_{1} / R_{O}$ 
is greater than the angular velocity $\omega_{2}=v_{2} / R$, then the rays of type 1 determine the asymptotic wave front. On the other hand, if $\omega_{2}>\omega_{1}$, then the rays of type 2 determine the asymptotic fronts. In other words, the asymptotic front is determined by the minimal loop. The exact formulation of the statement is Theorem 2 in Section 4. The envelope of the refracted rays forms a caustic, the refracted rays are tangential to the caustic in question.

3. Geometric wave theory in a heterogeneous medium. The considerations detailed in this section are valid, or can be easily generalized to a more general heterogeneous medium (where the velocity is piecewise constant), but we restrict ourselves to the case of the annular region $V$.

3.1. Properties of Fermat rays. In this subsection we deal with the structure of the Fermat rays (see Definition 6), and the fronts determined by them. The proofs of the following two simple lemmas are elementary.

Lemma 1. Every Fermat ray consists of the following four types of arcs:

1. A circular arc on $O$.

2. A circular arc on $I$.

3. A line segment in int $F$.

4. A line segment in int $S$.

Lemma 2. If a Fermat ray contains two arcs of different type, then these can join only in the following way (see Fig. 3):

a. Arc 4 joins to Arc 1 tangentially to $O$.

b. Arc 3 joins to Arc 2 tangentially to I.

c. Arc 4 joins to Arc 2 with the critical angle of total reflection.

d. Arc 3 joins to Arc 4 according to Snell's law of refraction $\left(v_{1} / v_{2}=\sin \alpha_{1} / \sin \alpha_{2}\right)$.

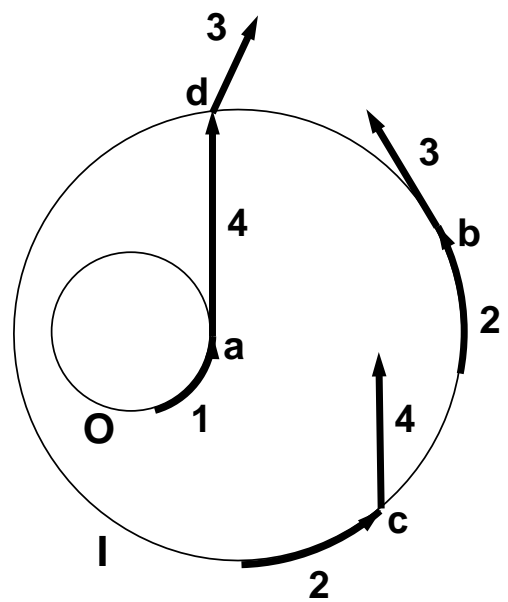

Figure 3: The arcs of Fermat rays and their combinations. 
In the construction of Fermat rays the caustics play an important role. A caustic is defined as the envelope of a special family of rays. According to Lemma 2, here three families of rays have importance:

A-rays: the rays departing tangentially outward from the obstacle,

B-rays: the rays departing tangentially outward from the interface,

C-rays: the rays departing inward from the interface with the angle of the total reflection $\left(\alpha_{\mathrm{cr}}\right)$.

For the tangential rays the caustic is just the borderline itself (obstacle or interface resp.). For the family of C-rays the caustic is a circle $K$ inside the interface centred at $C$ with radius $R / n$, where $n=v_{2} / v_{1}>1$ is the refractive index. Unfortunately the caustic $K^{\prime}$ of the rays of type 3 joining to rays of type 4 (which could be called D-rays, corresponding to Lemma $2 \mathrm{~d}$ ) is not circular, its shape is not discussed here.

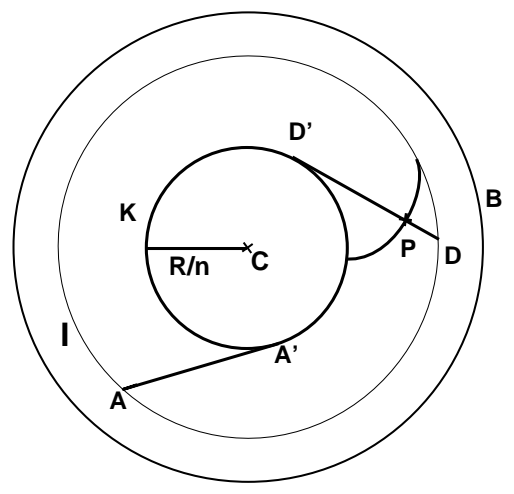

Figure 4: Use of caustic of the family of C-rays departing from the interface. The obstacle is not indicated here.

With the use of caustic $K$, the calculation of the propagation time of a heterogeneous path is an easy task and it has simple geometrical meaning. To formulate this let $A, D \in I$ and denote the tangent point on $K$ of the tangents from $A$ and $D$ by $A^{\prime}$ and $D^{\prime}$, see Fig. 4 . Let $P$ be a point of the segment $D D^{\prime}$. The propagation time of the real heterogeneous path arc $\overparen{A D}$ on the interface with velocity $v_{2}$ and segment $D P$ with velocity $v_{1}$ (shortly $\overparen{A D P}$ ) is related to that of a corresponding homogeneous path: tangential segment $P D^{\prime}$ and $\operatorname{arc} \overparen{D^{\prime} A^{\prime}}$ (anticlockwise on $K$ ) (shortly $P \overparen{D^{\prime} A^{\prime}}$ ) with the same velocity $v_{1}$. In exact terms

Proposition 1. Let $L=2 R \pi / n+A A^{\prime}$. Then for every point $P$ of the segment $D D^{\prime}$

$$
\tau(\overparen{A D P})=L / v_{1}-\tau\left(P \overparen{D^{\prime} A^{\prime}}\right)
$$

Proof.

$$
\begin{aligned}
& \tau(\overparen{A D P})=\overparen{A D} / v_{2}+D P / v_{1}=(\overparen{A D} / n+D P) / v_{1}=\left(\overparen{A^{\prime} D^{\prime}}+D P\right) / v_{1} \\
& \quad=\left(2 R \pi / n-\overparen{D^{\prime} A^{\prime}}+D D^{\prime}-P D^{\prime}\right) / v_{1}=\left(L-P \overparen{D^{\prime} A^{\prime}}\right) / v_{1}=L / v_{1}-\tau\left(P \overparen{D^{\prime} A^{\prime}}\right) .
\end{aligned}
$$

This way the use of caustic made it possible to convert the distances in the fast medium $F(\overparen{A D})$ into a distance in slow medium $S\left(\overparen{D^{\prime} A^{\prime}}\right)$. As a consequence of this 
proposition, one deduces that the wavefront generated by the C-rays is a reverse involute of $K$, see Fig. 4. A wavefront generated by C-rays is the locus of the points to which the propagation time on C-rays from a given point $(A)$ is constant. (The word reverse refers to the position of the involute [8], since the C-rays depart from the caustic in the negative (clockwise) direction, while A-rays generating also involute shape fronts depart from the obstacle in the positive direction.)

The next lemma is based on the following facts:

- The fronts are orthogonal to the rays.

- The orthogonal trajectories of the tangents of a curve are the involutes of the curve.

LEMMA 3.

1. The fronts generated by the family of $A$-rays are the involutes of $O$.

2. The fronts generated by the family of B-rays are the involutes of $I$.

3. The fronts generated by the family of $C$-rays are the reverse involutes of $K$.

DEFINITION 9.

1. Let $S_{O}$ be the obstacle generated part (zone) of $S$, that is the locus of the points in the neighbourhood of which $f_{t}$ is an involute of $O$.

2. Let $S_{I}$ be the interface generated part (zone) of $S$, that is the locus of the points in the neighbourhood of which $f_{t}$ is a reverse involute of $K$.

3. Let $F_{O}$ be the obstacle generated part (zone) of $F$, that is the locus of the points in the neighbourhood of which $f_{t}$ is a reverse involute of $K^{\prime}$.

4. Let $F_{I}$ be the interface generated part (zone) of $F$, that is the locus of the points in the neighbourhood of which $f_{t}$ is an involute of $I$.

5. Let $S_{b}$ be the boundary between $S_{O}$ and $S_{I}$.

6. Let $F_{b}$ be the boundary between $F_{O}$ and $F_{I}$.

THEOREM 1. For $t$ large enough, the sets $S$ and $F$ can be divided into three connected subsets $S=S_{O} \cup S_{I} \cup S_{b}, F=F_{O} \cup F_{I} \cup F_{b}$.

Moreover, $S_{O}\left(F_{I}\right)$ consists of tangential segments of $O(I)$, that is if $P \in S_{O}\left(F_{I}\right)$, then the entire tangential segment starting from $O(I)$ ending at $P$ is also contained in $S_{O}\left(F_{I}\right)$. Similarly, if $P \in S_{I}\left(F_{O}\right)$, then the entire tangential segment of $K\left(K^{\prime}\right)$ between $P$ and $I$ is also contained in $S_{I}\left(F_{O}\right)$.

Pr o of. We prove only the division of $S$, the case of $F$ is similar. Let $P \in \operatorname{int} S$, then the last arc of the Fermat ray reaching $P$ should be a line segment (of type 4 ). If $t$ is large enough then $Q$, the other endpoint of the segment cannot be on $f_{0}$, therefore it should be on $O$ or on $I$. It is easy to see that the arc of the Fermat ray before the segment $Q P$ is on $O$ (type 1 ) or on $I$ (type 2). Thus according to Lemma $2 Q P$ is an A-ray or a C-ray. Because of continuity the points in a small neighbourhood of $P$ are reached by the same type of rays as $P$. According to Lemma 3 the front $f_{t}$ (containing the point $P$ ) is an involute of $O$ (in this case $P \in S_{O}$ ), or a reverse involute of $K$ (in this case $P \in S_{I}$ ). The boundary $S_{b}$ consists of those points $P$ to which $Q$ can be both on $O$ and $I$, that is the points which can be reached on two different Fermat rays.

The fact that subsets $S_{O}, S_{I}, F_{O}$ and $F_{I}$ consist of tangential segments, follows from the simple statement that any part of a Fermat ray is also a Fermat ray. 
Thus we have proved that, similarly to the symmetric case, the rays departing from $O$ and $I$ will generate the asymptotic fronts, which consist of two parts: an inner part (generated by the rays departing from $O$ ), and an outer part (generated by the rays departing from $I$ ). (Some front lines may contain only one part.) The two front parts join each other at a 'break point' where their tangents are not the same in a generic case. In the next subsection we establish the rules governing the motion of the break point, in order to get more information on the structure of the inner and the outer zones (the sets $S_{O}, F_{O}$, and $\left.S_{I}, F_{I}\right)$.

3.2. Local dynamics of the break point. The following considerations are elementary, but they are necessary for studying the evolution of wave fronts in question. Since the motion of the break point is determined locally by a small part of the front in the neighbourhood of the break point, therefore we can assume that the two front parts (joining at the break point) are straight segments. The evolution of the break point depends on the angle (and not on the shape) of the two front parts.

3.2.1. Homogeneous medium. Assume that the velocity is constant in a neighbourhood of the break point. Two cases should be distinguished according to the convexity of the domain behind the front (see Fig. 5).

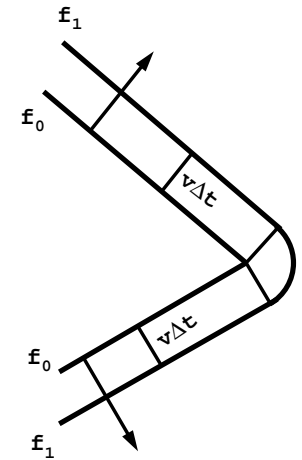

a.

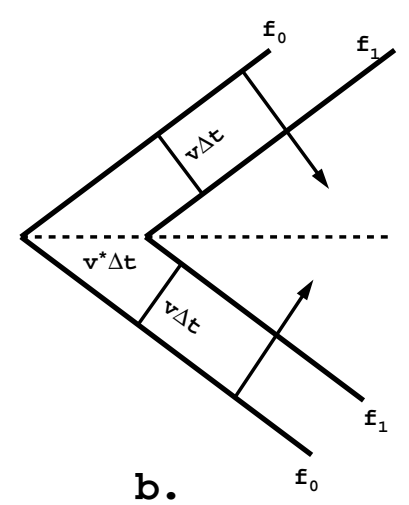

b.

Figure 5: Evolution of a broken front in a homogeneous medium.

(a) Convex case. The break point immediately ceases to exist, the two parts of the front will be connected smoothly by a circular arc due to the Huygens-principle.

(b) Concave case. In this case, the break point will move in the direction of the bisector.

Using Fermat's principle, or equivalently a symmetry consideration we get the following so called bisector rule for the motion of the break point.

Proposition 2 (Bisector rule).

1. In the convex case the break point ceases to exist, the two parts of the front will be connected smoothly by a circular arc.

2. In the concave case the front preserves its broken character, and the break point will move in the direction of the bisector. 
Note that break points can die or can be born at some exceptional points, which does not occur in our case. For the exact theory of the singularities of wave fronts see [2].

3.2.2. Heterogeneous medium. Now we investigate the motion of the break point located initially at a point of an interface of two homogeneous media. For this moment, we consider only the concave case. The motion of the break point depends on the virtual velocity of the two front parts. To define the notion of virtual velocity, let us consider a front, forming an angle $\alpha$ with a given boundary line, see Fig. 6 .

a.

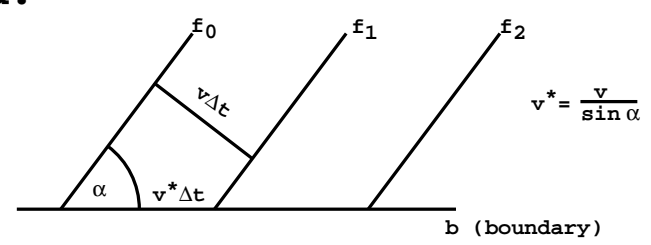

b.

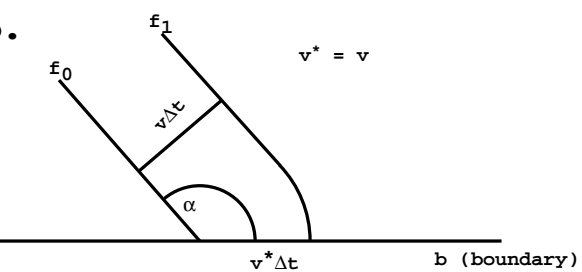

Figure 6: Virtual velocity at a boundary line. The common point of the front with the boundary will move with the speed $v^{*}$. (a) Acute angle, (b) Obtuse angle.

Definition 10. The virtual velocity of the front with respect to that boundary line is

$$
v^{*}= \begin{cases}v / \sin \alpha & \text { if } 0<\alpha \leq \pi / 2 \\ v & \text { if } \pi / 2<\alpha<\pi .\end{cases}
$$

Now consider a broken front, the break point of which is on the interface. Let us denote by $\alpha_{1}$ and $\alpha_{2}$ the angle of the front parts to the interface (see Fig. 7). These angles are equal to the angle of incident, that is the angle between the ray and the normal to the border line. The following proposition can be proved by elementary geometry.

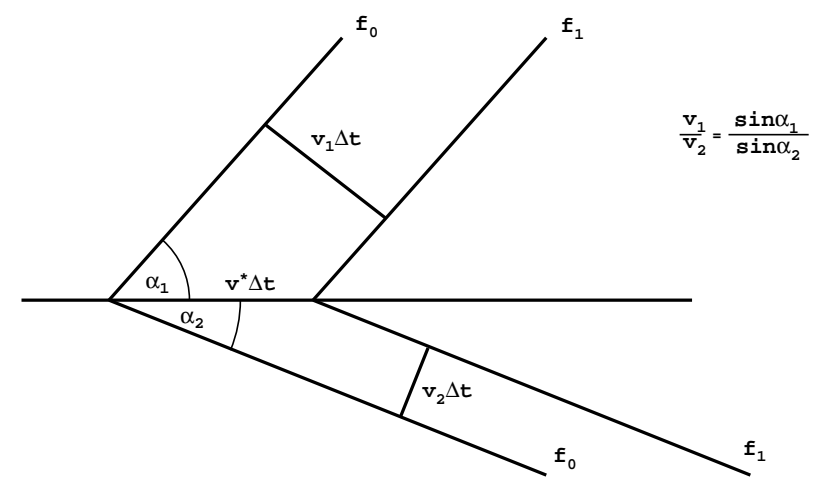

Figure 7: The break point remains on the interfacial boundary, provided that the virtual velocities for the two parts of the front are equal.

Proposition 3. The break point moves

- on the interface if virtual velocities of the front parts are equal, that is

$$
v_{1} / \sin \alpha_{1}=v_{2} / \sin \alpha_{2}
$$

- into the region where the virtual velocity of the front part is smaller. 
Thus the front part with the greater virtual velocity will cross the borderline and continues there according to Snell's law. The break point (between the incurred front part and the front part with smaller virtual velocity) will move inward the region in question following the bisector rule. Thus the boundary of the domains of the original front parts will move in such a way, that the domain possessing greater virtual velocity at the interfacial boundary, will increase. Several different patterns are possible depending on the propagation velocities and the angles of the initial front parts.

3.2.3. The break point portrait. From the general rules of local break point dynamics follow useful consequences for the special arrangement given in Definition 1 (see Fig. 1). According to Theorem 1 after enough long time the front $f_{t}$ consists of two parts in the set $S$ : an inner part which is the involute of $O$ and an outer part which is a reverse involute of $K$. A break point (moving in the set $S_{b}$ ) separates the two parts. The motion of the break point is determined by the bisector rule (Proposition 2) in the homogeneous region $S$.

However, an initial break point $P$ can be chosen arbitrarily in $S$. The position of the break point $P$ determines an involute of $O$ and a reverse involute of $K$ which contains $P$. The direction of the break point motion at $P$ is the bisector of the tangents of the involute and reverse involute at $P$. In this way we get a direction field (of break point motion) in $S$, which determines (apart from exceptional situations) a phase portrait. This will be referred to as "break point portrait". The trajectories on the break point portrait are the curves on which a break point can move.

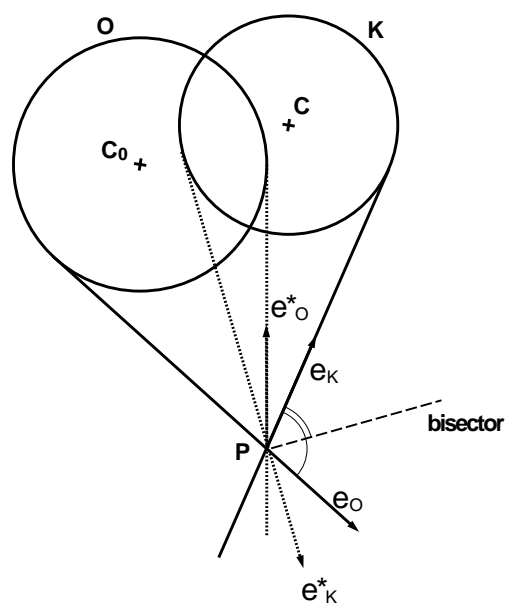

Figure 8: In this situation the rear part of $O$ stays behind that of $K$, and the forepart of $O$ stays behind that of $K$. In other terms, this means that for the "dual" tangential unit vector $e_{K}^{*}$ to $K$ and for the tangential unit vector $e_{O}$ to $O \arg \left(e_{O}\right)>\arg \left(e_{K}^{*}\right)$. Consequently, the bisector constitutes an acute angle with the radius $r$ to the point $C$. On the other hand, for the "dual" tangential unit vector $e_{O}^{*}$ and for the tangential unit vector $e_{K} \arg \left(e_{O}^{*}\right)>\arg \left(e_{K}\right)$. Consequently, the bisector constitutes an obtuse angle with the radius to the point $C_{O}$.

Now we establish the rules which determine whether the trajectory crosses the circles centred at $C$, resp. $C_{O}$, inward or outward. Let us introduce the tangent unit vectors from 
the break point $P$ to the obstacle $\left(e_{O}\right.$ and $\left.e_{O}^{*}\right)$ and to the caustic $\left(e_{K}\right.$ and $\left.e_{K}^{*}\right)$. More exactly, $e_{O}$ is the unit vector of the A-ray at point $P, e_{K}$ is the unit vector of the C-ray at point $P$. The other unit vectors tangent to $O$ and $K$ are denoted by $e_{O}^{*} e_{K}^{*}$ respectively. These notations corresponds to counterclockwise propagation. The break point $P$ moves in the direction of the bisector of $e_{O}$ and $e_{K}$, see Fig. 8.

\section{Proposition 4.}

1. The distance between the break point $P$ and $C$ decreases in time if and only if $\arg \left(e_{O}\right)>\arg \left(e_{K}^{*}\right)$. (Here $\arg$ is the usual term for the angle of a vector in polar coordinates.)

2. The distance between the break point $P$ and $C_{O}$ decreases in time if and only if $\arg \left(e_{O}^{*}\right)<\arg \left(e_{K}\right)$.

Pictorially, the distance of the break point $P$ from $C$ decreases if and only if the rear of $O$ stays behind that of $K$. Here the term 'stays behind' is used from the viewpoint of an observer moving counterclockwise at $P$. That is in Fig. 8 where $\arg \left(e_{O}\right)>\arg \left(e_{K}^{*}\right)$ the observer sees the rear of $O$ behind that of $K$. The distance of the break point $P$ from $C_{O}$ decreases if and only if the forepart of $O$ is before that of $K$.

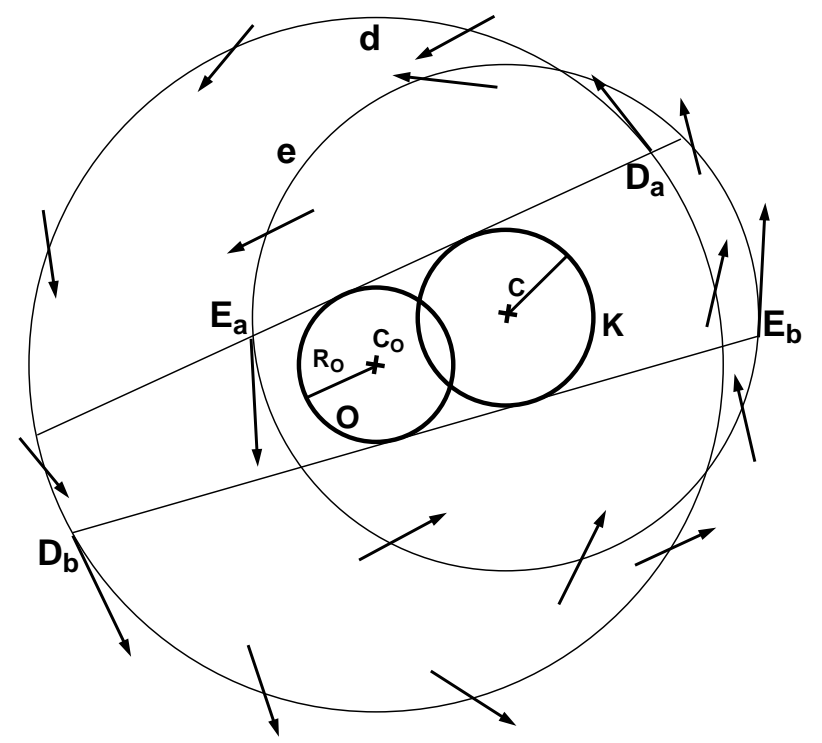

Figure 9: The break point moves inward in the arc $E_{a} E_{b}$, and moves outward in the arc $E_{b} E_{a}$ of the circle $e$ centred at $C$. On the other hand, it moves inward in the arc $D_{a} D_{b}$, and moves outward in the arc $D_{b} D_{a}$ of the circle $d$ centred at $C_{O}$.

These rules provide us information about the qualitative character of the "break point portrait". One can determine whether the break point moves inward or outward on a circle around $C$ and $C_{O}$. It is easy to see that the common tangents of $O$ and $K$ divide any circle (centred at $C$ or $C_{O}$ respectively) into two parts: the break point moves inward in one part, and outward on the other part. In Fig. 9 there are two circles $(e$ is centred at $C, d$ is centred at $C_{O}$ ); the arrows show the direction of the break point motion. These 
characteristics of the local break point dynamics can be used to construct the global portrait similarly to the use of the Lyapunov functions in dynamical systems theory [6].

4. Classification according to the global properties. In general the domain of the obstacle-generated front parts and the interface-generated front parts may be located differently, and accordingly to those locations many different classes can be distinguished depending on the geometric data as well as on the ratio of the velocities. For short, we will not list all the possible types, our aim is to illustrate the peculiarities of the asymmetric arrangement.

Three main classes will be termed as weak, moderate and strong asymmetric cases.

DeFinition 11. The reactor $V$ is called

1. weakly asymmetric, if either the caustic is inside the obstacle or the obstacle is inside the caustic;

2. moderately asymmetric, if the caustic intersects the obstacle;

3. strongly asymmetric, if the caustic and the obstacle are disjoint sets (and do not contain each other).

If the caustic $(K)$ is inside the obstacle, then the minimal loop is the interface and the obstacle-generated domain is empty. On the other hand, if the obstacle is inside the caustic, then the minimal loop is the obstacle, and the interface-generated domain is empty. These cases are qualitatively equivalent to the corresponding symmetric arrangements [8]. That is, for the weakly asymmetric case we have (after elementary calculations):

THEOREM 2 (Weak asymmetry).

1. If $K$ is inside $O$, then $S_{O}=F_{O}=\emptyset, S_{I}=S$ and $F_{I}=F$.

2. If $O$ is inside $K$, then $S_{I}=F_{I}=\emptyset, S_{O}=S$ and $F_{O}=F$.

While in the case of weak asymmetry the minimal loop is either the obstacle or the caustic, in the case of moderate or strong asymmetry the minimal loop may be of a third type, a mixed one. If the caustic and the obstacle have common tangents, and these common tangents do not intersect each other inside $S$ in the direction of the obstacle, then we can construct a loop $M$ in such a way that it consists of an $\operatorname{arc}$ of $O$, an $\operatorname{arc}$ of $I$ and two segments of the common tangents (see Fig. 10).

Definition 12. The simple closed curve $M$ is called mixed loop if it consists of the following four arcs (see Fig. 10): the arc $O_{b} O_{a}$ of the obstacle; the segment $O_{a} I_{a}$; the arc $I_{a} I_{b}$ of the interface; the segment $I_{b} O_{b}$.

To classify the different types of the break point portraits, it is important to know the minimal loop. From now on we shall assume that the reactor $V$ is moderately asymmetric, the strongly asymmetric case will not be considered here. We also assume that the propagation time belonging to the obstacle, the interface and the mixed loop are different. 


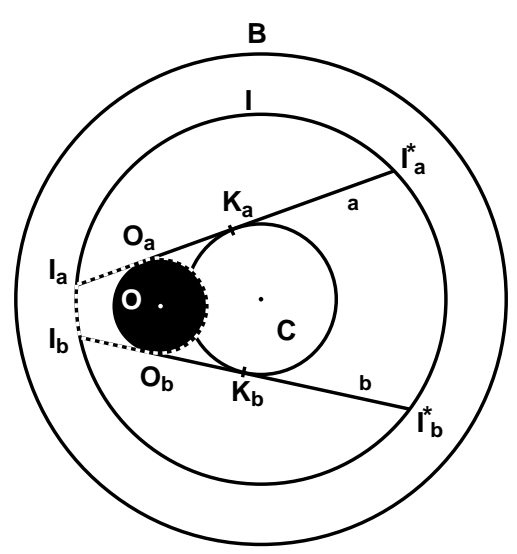

Figure 10: Construction of the mixed loop $M$. This simple closed curve $M$ can be defined if and only if the common tangents exist (moderate or strong asymmetry), and they do not intersect each other inside $S$ in the direction of the obstacle (in the present case on the left side).

Proposition 5. The minimal loop is either the obstacle, or the interface, or the mixed loop.

Proof. It is easy to see that if the minimal loop has no point in int $S$, then the minimal loop is the obstacle or the interface. If it has a point $P \in \operatorname{int} S$, then the arc of the minimal loop containing $P$ is a segment the endpoints of which are on $O$ and $I$. According to Lemma 2 this segment is tangential both to $O$ and $K$. Hence the arcs of the minimal loop are on $I, O$ and on the common tangents of $O$ and $K$. Comparing the propagation time of the remaining candidates for the minimal loop, one gets that the minimal loop is a mixed one.

In the evolution of the front we can distinguish three stages:

- First stage: Starting from the initial front, rays are emanated perpendicularly, one or two leading points appear. At the end of this stage the front consists of an obstacle and an interface generated part (maybe only one of them).

- Second stage: Since the front consists of two parts, the rules of break point dynamics are applied. In our case the break point dynamics determines the motion of the break point uniquely. Hence, if the front consists of at most two parts (obstacle-generated and interface-generated part), then all the later fronts possesses this property. Break points can be born at the obstacle and at the interface, and can die at the obstacle or at the outer boundary. At the end of this stage the zones take their final form.

- Third stage: Periodic motion of the varying shaped front around the obstacle. The break point moves along the boundary of the zones $\left(S_{b}\right.$ and $\left.F_{b}\right)$. This break point trajectory will be referred to as stationary break point curve. This curve may have different directed branches.

To determine the asymptotic front shapes we use the following tools: break point dynamics, comparison of the propagation times belonging to the two zones. The propagation times for the two zones must equal at the boundary. 
Using these tools the following propositions can be proved with lengthy but straightforward consideration. As an illustration we outline only the proof of Proposition 7.

Proposition 6. The starting point of a stationary break point curve can be the point $O_{b}$ of the obstacle; or the point $I_{a}$ of the interface (see Fig. 10).

If the starting point is $O_{b}$, then the first part of the arc is the straight segment $O_{b} K_{b}$.

If the starting point is $I_{a}$, then two break point curve start here, one of them is inside $S$, the other one is a straight line tangential to $I$.

Proposition 7. If a stationary break point branch starts from $O_{b}$ (see Fig. 10), then the minimal loop is not the obstacle. If this branch reaches the interface, then the minimal loop is $M$, while if it ends at another point of $O$, then the minimal loop is $I$.

Pr o of. If a stationary break point branch starts from $O_{b}$, then the front $f_{t}$ containing the point $O_{b}$ is the reverse involute of $K$ at $O_{b}$. From Proposition 4 follows that the break point moves first in the straight segment $O_{b} K_{b}$, then it moves outside $K$ in the region above the line $K_{b} I_{b}^{*}$. Since the stationary break point branch starts from $O_{b}$, there are two possibilities:

i) The break point crosses $I$ somewhere between $I_{b}^{*}$ and $I_{a}$. Then the (entirely obstacle generated) front moves further and a new break point appears at $I_{a}$ moving downward and inward. If it crossed the segment $I_{b} O_{b}$, then $O_{b}$ would not be a break point in the next round, which contradicts to the assumption that the stationary break point branch starts from $O_{b}$. Hence the new break point ends at $O$ before $O_{b}$, then - as it can be seen-neither $O$ nor $I$ is the minimal loop.

ii) The break point reaches $O$ at a point $O_{1}$ before $O_{b}$. Then the front at $O\left(O_{1}\right)$ is a reverse involute of $K$, its common point with $K$ and $I$ is denoted by $K_{0}\left(K_{1}\right)$ and $I_{0}\left(I_{1}\right)$, respectively. It is easy to see that the length of the $\operatorname{arc} O_{1} O_{b}$ is greater than the corresponding arc $K_{1} K_{0}$ on the caustic $K$. Since the propagation time on the arc $I_{0} I_{1}$ is equal to the propagation time of $O_{b} O_{1}$, therefore the propagation time of $O$ $\left(=O_{b} O_{1}+O_{1} O_{b}\right)$ is greater than that of $I\left(=K_{0} K_{1}+K_{1} K_{0}\right)$.

Proposition 8. If a stationary break point branch starts from $I_{a}$ (see Fig. 10), then the minimal loop is not the interface. If it reaches $O$, then another branch must start from $O_{b}$ and the minimal loop is $M$. If it does not reach $O$, then it reaches $I$ at another point (beyond $I_{b}^{*}$ ) and the minimal loop is $O$.

Now we are in the position to solve our problem (determine the shape of the stationary fronts) in the case of moderate asymmetry. According to Theorem 1 it is enough to determine the different zones $\left(S_{O}, S_{I}, F_{O}, F_{I}\right)$. The structure of these zones depends on the minimal loop, hence we have three different cases. Using the above propositions one gets the following statements.

1. If the minimal loop is $I$ (Fig. 11), then the leading point will rotate in $I$ without any trouble. Thus $F=F_{I}$ (hence $F_{O}=\emptyset$ ), and $S_{I}$ contains a neighbourhood of $I$. However, 
there is a zone in which the rays emanating from $O_{b}$ determine the fronts, that is $S_{O} \neq \emptyset$, but this zone is inside $S$ as an "enclave", that is $\overline{S_{O}} \subset S$.

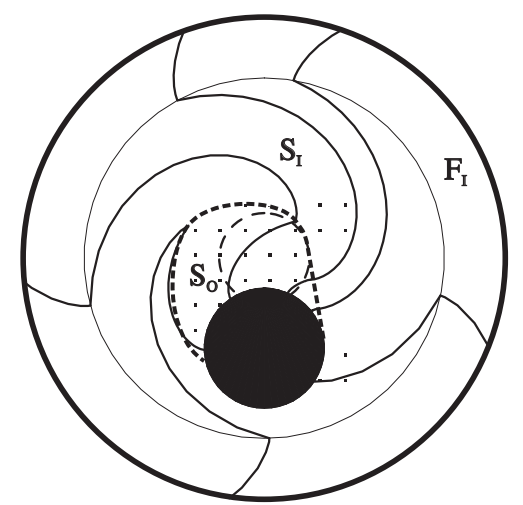

Figure 11: Stationary fronts and zones. The obstacle generated zone $\left(S_{O}\right)$ is dotted. Case: minimal loop is the interface.

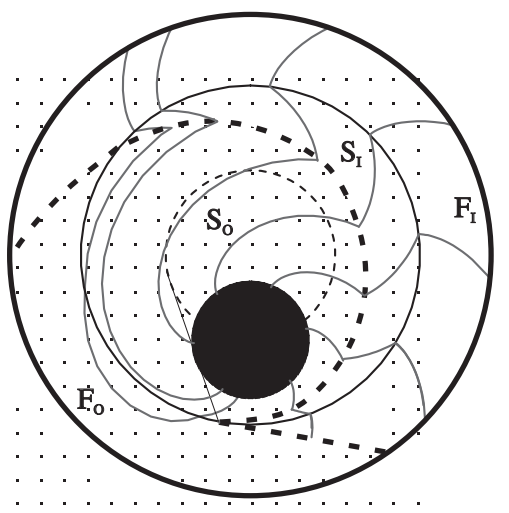

Figure 12: Stationary fronts and zones. The obstacle generated zone $\left(S_{O} \cup F_{O}\right)$ is dotted. Case: minimal loop is the obstacle.

2. If the minimal loop is $O$ (Fig. 12) then the zone $S_{O}$ engulfs $O$ totally. The leading point is rotating periodically on $O$. However, a break point appears at $I_{a}$, and from that another leading point generates the fronts in the "enclave" $S_{I}$. The nonempty zones $F_{I}$ and $F_{O}$ are shown in Fig. 12.

3. If the minimal loop is $M$ (Fig. 13), then both $S_{O}$ and $S_{I}$ are nonempty "enclaves": the leading points in $O$ and $I$ are not able to make entire rounds, because the other leading point ignites somewhere before along its path. The nonempty zones $F_{I}$ and $F_{O}$ are shown in Fig. 13.

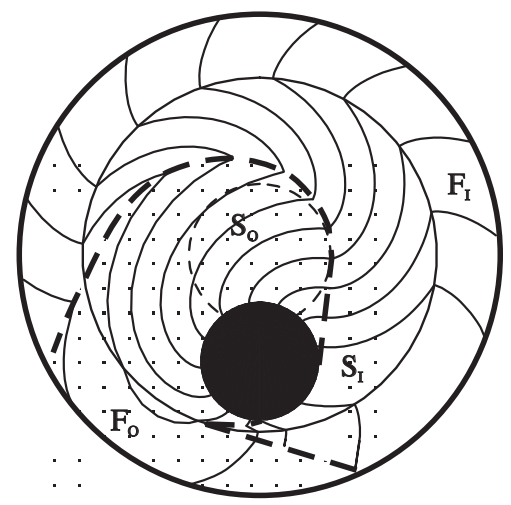

Figure 13: Stationary fronts and zones. The obstacle generated zone $\left(S_{O} \cup F_{O}\right)$ is dotted. Case: minimal loop is the mixed loop.

Acknowledgments. We thank Professors D. Siersma, V. Zakalyukin, S. Janeczko and Z. Noszticzius for discussion. 


\section{References}

[1] V. I. Arnol'd, Mathematical Methods of Classical Mechanics, Springer, New York, 1978.

[2] V. I. Arnol'd, Singularities of Caustics and Wave Fronts, Math. Appl. (Soviet Ser.) 62, Kluwer Academic Publ., Dordrecht, 1990.

[3] H. Farkas, I. Farago and P. L. Simon, Qualitative properties of conductive heat transfer, in: Thermodynamics of Energy Conversion and Transport, S. Sieniutycz and A. De Vos (eds.), Springer (to appear).

[4] H. Farkas, I. Mudri, Shape-preserving time-dependences in heat conduction, Acta Phys. Hungar. 55 (1984), 267-273.

[5] P. C. Fife, Mathematical Aspects of Reacting and Diffusing Systems, Lecture Notes in Biomath. 28, Springer, Berlin, 1979.

[6] J. Guckenheimer, Ph. Holmes, Nonlinear Oscillations, Dynamical Systems, and Bifurcations of Vector Fields, Appl. Math. Sci. 42, Springer, New York, 1983.

[7] S. Janeczko, I. Stewart, Symplectic singularities and optical diffraction, in: Singularity Theory and its Applications, Part II, M. Roberts and I. Stewart (eds.), Lecture Notes in Math. 1463, Springer, Berlin, 1991, 220-255.

[8] A. Lázár, H. D. Försterling, H. Farkas, P. L. Simon, A. Volford, Z. Noszticzius, Waves of excitations on nonuniform membrane rings, caustics, and reverse involutes, Chaos 7 (1997), 731-737.

[9] A. Lázár, Z. Noszticzius, H. Farkas, H. D. Försterling, Involutes: the geometry of chemical waves rotating in annular membranes, Chaos 5 (1995), 443-447.

[10] J. D. Murray, Mathematical Biology, Biomathematics 19, Springer, Berlin, 1989.

[11] Z. Noszticzius, W. Horsthemke, W. D. McCormick, H. L. Swinney, W. Y. Tam, Sustained chemical waves in an annular gel reactor: a chemical pinwheel, Nature 329 (1987), 619-620.

[12] J. Sainhas, R. Dilão, Wave optics in reaction-diffusion systems, Phys. Rev. Lett. 80 (1998), 5216-5219.

[13] S. K. Scott, Oscillations, Waves and Chaos in Chemical Kinetics, Oxford University Press, Oxford, 1994.

[14] S. Sieniutycz, H. Farkas, Chemical waves in confined regions by Hamilton-JacobiBellman theory, Chemical Engineering Science 52 (1997), 2927-2945.

[15] P. L. Simon, H. Farkas, Geometric theory of trigger waves. A dynamical system approach, J. Math. Chem. 19 (1996), 301-315.

[16] N. Wiener, A. Rosenblueth, The mathematical formulation of the problem of conduction of impulses in a network of connected excitable elements, specifically in cardiac muscle, Arch. Inst. Cardiol. México 16 (1946), 205-265. 